\title{
HOALDI - um conjunto de heurísticas para a avaliação e desenvolvimento de Objetos de Aprendizagem para Lousa Digital Interativa
}

\author{
Henrique de A. Silva, \\ Ana Carolina G. Inocêncio \\ Curso de Ciências da Computação \\ Universidade Federal de Goiás (UFG) \\ Regional Jataí \\ Caixa Postal 01 - CEP 75.801 - 615 \\ Jataí - GO - Brasil \\ henriarasilva@gmail.com \\ anacarolina.inocencio@gmail.com
}

\begin{abstract}
Interactive Whiteboard (IWB) has been shown to be a technology that has brought interactivity to the teaching-learning process, but some Learning Objects (LOs) present usability problems with this technology. Therefore, this paper aims to propose an efficient set of heuristics for the development and evaluation of LOs, adapting them to IWB. For this, a review was made of the literature on the usability of IWB and LOs quality, as well as a practical exploration of it. After the experiment, the HOALDI set proved to be efficient to be used in the evaluation of LOs in IWB.
\end{abstract}

Resumo. A Lousa Digital Interativa (LDI) tem se mostrado uma tecnologia que trouxe interatividade para o processo de ensino-aprendizagem, porém alguns Objetos de Aprendizagem (OAs) apresentam problemas de usabilidade em relação a esta tecnologia. Sendo assim, o objetivo deste trabalho é propor um conjunto eficiente de heurísticas para desenvolvimento e avaliação de OAs, adequando-os a LDI. Para isto, foi feita uma revisão da literatura sobre a usabilidade da LDI e qualidade de OAs, bem como uma exploração prática da mesma. Após o experimento, o conjunto HOALDI se mostrou eficiente para ser usado na avaliação de OAs na LDI.

\section{Introdução}

A Lousa Digital Interativa (LDI) é uma tecnologia que utiliza de Objetos de Aprendizagem (OAs) para o auxílio no processo de ensino-aprendizagem. Um OA possui como característica principal a reusabilidade (Audino e da Silva Nascimento, 2010), (Braga et al., 2012) e (Tarouco et al., 2011). Apesar dos OAs terem como principal característica a reusabilidade, um problema é que grande parte deles não são desenvolvidos visando a dinâmica da LDI ${ }^{1}$ (Fiscarelli et al., 2016) e (Kalinke e Mocrosky, 2015). Sendo assim, o

\footnotetext{
${ }^{1}$ A dinâmica da LDI refere-se a forma de interação da LDI e como o usuário manuseia e tem acesso aos seus recursos
} 
VII Congresso Brasileiro de Informática na Educação (CBIE 2018)

Anais dos Workshops do VII Congresso Brasileiro de Informática na Educação (WCBIE 2018)

objetivo geral deste trabalho é proporcionar um melhor uso da LDI, de forma a possibilitar a criação/utilização de OAs que otimizem o uso dos recursos disponibilizados pela LDI, adaptando a sua dinâmica.

Os métodos de avaliação já existentes para OAs na literatura, como os de (Fiscarelli et al., 2016) (Mussoi et al., 2010) e (Silveira e Carneiro, 2012), apresentam várias limitações que, consequentemente, podem prejudicar a avaliação dos OAs. Assim, verificou-se a necessidade da criação de um novo conjunto de heurísticas, que foi denominado HOALDI (Heurísticas para a Avaliação e Desenvolvimento de Objetos de Aprendizagem para a Lousa Digital Interativa). Esse conjunto de heurísticas tem por intenção avaliar características relacionadas a interface, conteúdo, multimídia, elementos educacionais e a manuseabilidade dos OAs.

O conjunto HOALDI é descrito de forma objetiva, com a intenção de que os avaliadores, que não são especialistas, possam utilizá-lo na avaliação de OAs, possibilitando uma avaliação detalhada para verificar se o OA está apto a ser inserido no ambiente educacional em conjunto com a LDI.

\section{Lousa Digital Interativa}

A LDI é um sistema de exibição interativo comumente usado em aplicações educacionais. A LDI estabelece relação entre a superfície de ensino (um grande painel de parede, sensível à toques), um projetor e um computador digital. A superfície de ensino permite ao usuário operar o computador por meio da interação com a imagem projetada. Devido a eficiência durante o processo de ensino-aprendizagem houve um aumento considerável no número de LDIs instaladas nas escolas do mundo, porém os alunos não conseguem utilizar todos os recursos de um OA na LDI, isso baixa muito sua usabilidade (Navarro e Kalinke, 2016) e (Aytaç, 2013).

\section{Avaliação Heurística}

A Avaliação Heurística (AH) trata-se de um método de inspeção de usabilidade conduzido por 3 a 5 avaliadores (especialistas e não especialistas) geralmente, de forma que é utilizado um conjunto de heurísticas e avaliado se uma interface de software inspecionado obedece as heurísticas ou não (Nielsen, 1994). A gravidade das consequências de cada heurística infrigida é julgada pelo avaliador, o que sinaliza a necessidade ou conveniência de se alterar a interface para tornar o grau de usabilidade dela maior (de Castro Salgado et al., 2006). De acordo com Nielsen (1994), as gravidades podem ser julgadas com valores de 0 a 4 ou de 1 a 5 .

\section{Trabalhos Relacionados}

Durante o mapeamento sistemático realizado (Silva et al., 2017), o trabalho de Fiscarelli et al. (2016) (FCHECKLIST) foi o único trabalho encontrado que apresentou um método para avaliação de OAs na LDI (por meio de uma checklist).

\section{Heurísticas para Objetos de Aprendizagem na Lousa Digital Interativa}

O conjunto de heurísticas deste trabalho, denominado Heurísticas para Objetos de Aprendizagem na Lousa Digital Interativa (HOALDI), é apresentado como alternativa 
VII Congresso Brasileiro de Informática na Educação (CBIE 2018)

Anais dos Workshops do VII Congresso Brasileiro de Informática na Educação (WCBIE 2018)

a FCHECKLIST, partindo de uma fundamentação mais sólida. Para isso, o conjunto HOALDI foi fundamentado em: i) experiências de especialistas na área de computação e educação; ii) trabalhos que abordam características de um bom OA e como ter uma boa usabilidade, tanto da LDI quanto de OA; ii) trabalhos relacionados que também fizeram de alguma forma validação para OAs. A seguir estão as heurísticas que foram criadas para o conjunto HOALDI para os aspectos pedagógicos, de manuseio e técnicos.

- Aspectos Pedagógicos: HP1 - Usuário recebe um feedback direto ou indireto a cada ação; HP2 - Há alguma opção de ajuda, que auxilia principalmente usuários iniciantes; HP3 — Objetivo é autoexplicativo, sendo posto de forma clara e impulsionando o usuário a atingí-lo; HP4 — Há correspondência entre elementos do mundo real e do ambiente; HP5 - O usuário tem facilidade em reconhecer funções para executá-as; HP6 — Linguagem utilizada clara e adequada ao público-alvo; HP7 - Contém pouco texto para explicação dos conteúdo da disciplina, sendo possível o usuário aprender pela observação do fluxo de atividades; HP8 - Os elementos educacionais estão organizados separadamente com fim específico; HP9 - Os aspectos da disciplina que se pretende ensinar estão corretos e coerentes; HP10 - Há uma recompensa ou outra forma de incentivo para motivar o aluno a continuar aprendendo e utilizando o OA;

- Aspectos de Manuseio: HM1 - $O$ design do OA não chama a atenção em elementos que não são importantes para a execução de atividades; HM2 - A combinação de cores não atrapalha ou incomoda a visibilidade; HM3 — Os controles são bem visíveis, deixando claro a forma que o usuário deve interagir; HM4 - Possui affordance, o que facilita o reconhecimento de ações ao usuário; HM5 - São combinados vários elementos (imagens, textos, animações) a fim de tornar o OA mais atrativo de ser utilizado; HM6 - Os elementos estão posicionados de forma adequada, indicando ou deixando claro quais são manipuláveis; HM7 - O OA não utiliza de outros periféricos do computador, com exceção do mouse; HM8 - A resolução da imagem é suficiente para que a imagem fique de boa qualidade e não atrapalhe as atividades; HM9 - O OA promove ações em conjunto, de modo a unir professores e alunos; HM10 - O usuário consegue visualizar e acompanhar suas ações tranquilamente; HPM1 - Os elementos estão posicionados de forma adequada, indicando ou deixando claro quais são manipuláveis e a forma de manipular os mesmos; HM12 - Não exige-se um grande esforço o qual pode prejudicar o usuário a atingir seu objetivo, ou mesmo executar ações de forma atrasada com relação a outros dispositivos;

- Aspectos Técnicos: HT1 - Seu tamanho não passa de 4MB; HP2 - Carregamento das funcionalidades é rápido; HP3 - É reutilizável, de forma que seja possibilitado o seu adequado a qualquer dispositivo; HT4 - Não apresenta erros/bugs durante a execução.

\subsection{Formulação de hipóteses}

As hipóteses criadas buscam verificar qual trabalho (conjunto HOALDI ou FCHECKLIST) apresenta uma maior eficiência. A Tabela 1 demonstra as duas hipóteses formuladas. 
VII Congresso Brasileiro de Informática na Educação (CBIE 2018)

Anais dos Workshops do VII Congresso Brasileiro de Informática na Educação (WCBIE 2018)

Tabela 1. Hipóteses do Estudo Avaliativo

\begin{tabular}{|c|c|c|c|c|} 
H0 & $\begin{array}{l}\text { Não há diferença entre a eficiência para identificação de } \\
\text { problemas em um OA, seja utilizando o conjunto HOALDI } \\
\text { ou o FCHECKLIST, então EHOALDI = EFCHECKLIST } \\
\text { Há diferença entre a eficiência para identificação de } \\
\text { problemas em um OA, seja utilizando o conjunto HOALDI } \\
\text { ou o FCHECKLIST, então EHOALDI \#EFCHECKLIST }\end{array}$ \\
H0
\end{tabular}

\subsection{Planejamento da Avaliação}

Foi feita a distribuição de dois grupos de avaliadores, seguindo o roteiro padrão de uma AH, como especifica Nielsen (1994), cada grupo contendo quatro pessoas. A aplicação dos conjuntos HOALDI e FCHECKLIST foram realizadas por alunos de graduação, sendo estes classificados como avaliadores não especialistas. Também participaram especialistas, professores de graduação, que foram selecionados para avaliar o quão corretos estão os resultados obtidos pelos avaliadores não especialistas.

O OA Jogo do Sistema Solar do Cambito foi escolhido para a avaliação por estar disponível de forma gratuita na Internet, no repositório Escola Digital ${ }^{1}$ e possuir uma grande manuseabilidade, ou seja, é preciso clicar, arrastar e possuir grande aceitação pelos usuários, assim demonstrando que possui um nível de qualidade adequado para ser utilizado. O OA em questão, trata-se de um OA sobre planetas, em que o objetivo é colocá-los em ordem no Sistema Solar, de acordo com a proximidade que cada um tem do Sol. O OA atribui e retira pontos de acordo com o acerto ou erro do usuário. Após todas as etapas concluídas, o usuário pode clicar nos planetas e obter informações sobre eles. A Figura 1 representa uma captura de tela do OA em questão.

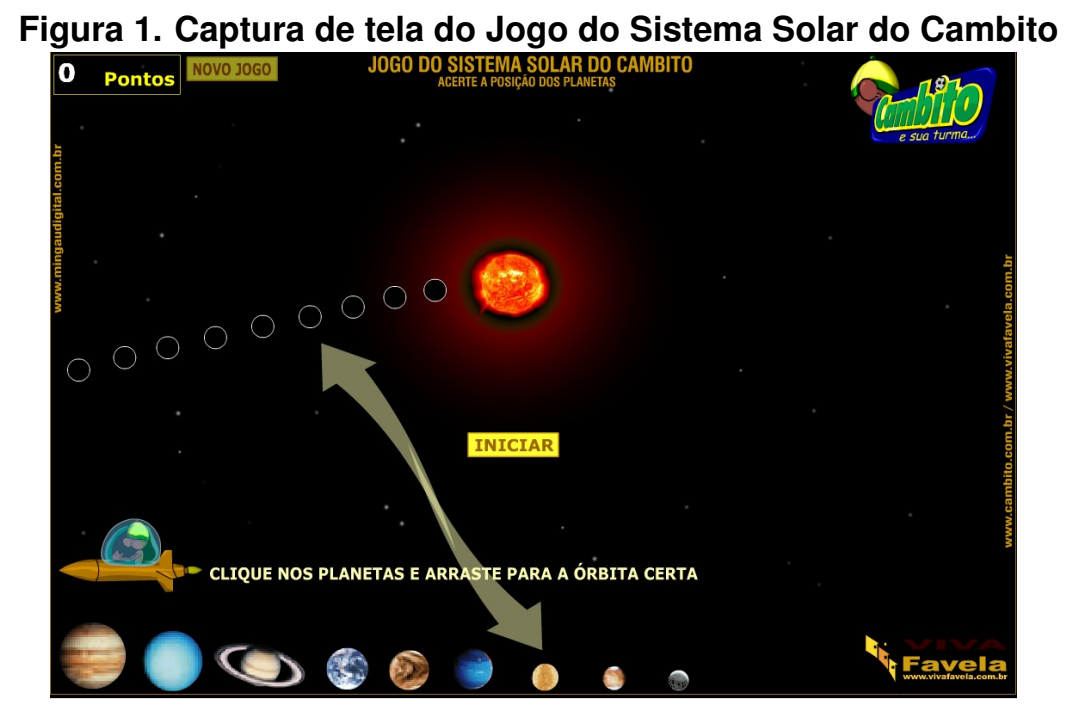

Foi verificada, por meio da aplicação de um questionário de caracterização de perfil, a experiência de cada avaliador, tanto especialistas quanto não especialistas, com questões relacionadas a $\mathrm{AH}$, OAs e Educação. E também foi aplicado um formulário referente ao Jogo do Sistema Solar do Cambito, sobre a carga educativa, estratégia educacional, medida preventiva, interface, e manipulação de elementos no OA. E posteriormente

\footnotetext{
${ }^{1}$ Repositório disponível em http://www.escoladigital.org.br
} 
VII Congresso Brasileiro de Informática na Educação (CBIE 2018)

Anais dos Workshops do VII Congresso Brasileiro de Informática na Educação (WCBIE 2018)

outro questionário sobre o método de avaliação utilizado. Os documentos utilizados para a realização da avaliação foram: Formulário de caracterização de perfil — O objetivo deste documento foi aplicar peguntas referentes à experiência e conhecimentos relevantes do avaliador, para que equilibrar os conhecimentos de cada equipe, de forma a não prejudicar a avaliação. Ele contém perguntas sobre experiência na Área da Educação, Avaliação Heurísticas e OAs; Formulário de Método de Avaliação - Divide-se em dois métodos, conjunto HOALDI e conjunto FCHECKLIST, cada um desses métodos foi utilizado por uma equipe. Este formulário contém perguntas sobre o OA utilizado; Formulário de Avaliação do Método de Avaliação - Neste formulário, os avaliadores atribuíram uma nota ao método de avaliação utilizado, considerando cada aspecto do mesmo, atribuindo uma nota de uma escala de 1 a 5 .

O projeto do estudo avaliativo foi composto das seguintes fases: FASE I: o OA foi avaliado por dois grupos de usuários não especialistas: Grupo HOALDI e Grupo FCHECKLIST; FASE II: O mesmo OA foi avaliado por dois grupos, desta vez de usuários especialistas: Grupo HOALDI' e Grupo FCHECKLIST'. Foi feito isso para que, posteriormente, os resultados tanto dos avaliadores especialistas quantos aos dos não especialistas fossem confrontados, para que se pudesse verificar qual método de avaliação é mais eficiente, principalmente, quando avaliadores não especialistas são consultados.

\subsection{Métricas utilizadas para análise dos dados coletados}

A qualidade das heurísticas criadas está diretamente relacionada ao quão próximo está o grau de severidade atribuído pelos usuários especialistas quanto aos do não especialistas (no caso do conjunto HOALDI). É importante essa comparação pois deve-se sempre considerar que a avaliação foi conduzida por não especialistas, e pode ser que esses avaliadores tenham identificado problemas com grau de severidade maior ou menor do que deveria ser. O cálculo das variáveis Eнодеdi Е Еснескціsт é dados pelas Fórmula (1) е (2), respectivamente, conforme a Tabela 2. Eнодеd e Еғснескцist variam de 0 zero a cem por cento. Quanto mais próximos dos 100\%, significa que mais coerentes são com os graus de severidade dados pelos avaliadores especialistas foram os graus dados pelos avaliadores não especialistas. A Tabela 2 demonstra a fórmula para calcular a eficiência dos conjuntos.

Tabela 2. Fórmula para calcular EнодLI e EFСнескLIST

(1) Se GSEhoaldi $<=$ GSNEhoaldi, então

$\mathrm{E}_{\text {HоALdi }}=\left(\mathrm{GSE}_{\text {HOALdi }} / \mathrm{GSNE}\right.$ HоALdi $\left.^{*} 100\right)$,

Senão

$\mathrm{E}_{\text {HоALdi }}=\left(\mathrm{GSNE}_{\text {HoALdI }} / \mathrm{GSE}_{\text {HоALdi }} * 100\right)$

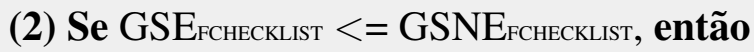

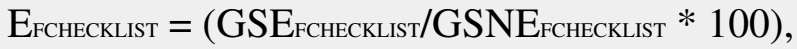

Senão

$\mathrm{E}_{\text {FCHECKLIST }}=\left(\mathrm{GSNE}_{\mathrm{FCHECKLIST}} / \mathrm{GSE}_{\mathrm{FCHECKLIST}} * 100\right)$

Fazendo uma suposição, em que a média dos graus de severidade dos avaliadores dos grupos A e B para a categoria "Estratégia Educacional" de OA, tenham valores iguais a 3.2 e 2.2, respectivamente, então GSNEнодdi (Média dos graus de severidade dados pelos avaliadores não especialistas que usaram o conjunto HOALDI) $=3.2$ e GSNEFснескLIST (Média dos graus de severidade dados pelos avaliadores não especialistas que usaram 
o conjunto FCHECKLIST) $=2.2$. No entanto, outros dois grupos de avaliadores A' e B', especialistas, chegaram às seguintes médias de grau de severidade para a categoria "Estratégia Educacional" do mesmo OA: GSEнодdi (Média dos graus de severidade dados pelos avaliadores especialistas que usaram o conjunto HOALDI) $=3.0$ e GSEFснескцIsт (Média das porcentagens, isto é, o quanto os avaliadores deram a mesma respostas na mesma classificação, transformadas em graus de severidade dados pelos avaliadores especialistas que usaram o conjunto FCHECKLIST) $=0.9$. Sendo assim, EHoaLdi $=93,75 \%$ e Е Еснескціsт $=40,90 \%$, ou seja, neste caso, os resultados obtidos pelos avaliadores não especialistas do Grupo A (que utilizaram o conjunto HOALDI), ficou mais próximo dos resultados dos especialistas, quando foram comparados aos resultados do Grupo B.

Para reforçar a validade do presente trabalho, também é utilizado o GQM (Goal, Metric, Question). As três etapas para a execução do método GQM são (de Souza et al., 2009): (i) Objetivo: listar os objetivos desejados; (ii) Questão: obter de cada objetivo as peguntas que serão respondidas, para ser possível constatar se o objetivo foi alcançado; (iii) Métrica: nesta etapa, deve-se decidir o que deverá ser medido (o que será avaliado) para que seja possível responder às perguntas criadas na etapa Questão.

Os objetivos para a validação dos métodos de avaliação de OAs por meio do GQM, levam em consideração três atributos, sendo eles: objetividade, tempo de avaliação e os problemas sem classificação. No conjunto FCHECKLIST entende-se como "classificação" os Indicadores da checklist, e no método de avaliação proposto neste trabalho refere-se às Heurísticas. Os três atributos são especificados a seguir.

- OBJETIVO de AVALIAÇÃo 1 - Análise: Método de Avaliação de OAs para a LDI; Para: Avaliação; Quanto à: Objetividade; Na visão: Dos professores e alunos; No contexto: Da sala de aula; Objetivo 1: Verificar se o Método de Avaliação possui Objetividade; Questão 1: As classificações são descritas de forma clara?; Métricas para realização da Questão 1: M1.1 - A forma da escrita está coerente, sem deixar dúvidas quanto ao seu significado; M1.2 - O texto não apresenta palavras ou termos muito genéricos, que podem dificultar o entendimento; Questão 2: As classificações não permitem múltiplas interpretações?; Métricas para realização da Questão 2: M2.1 - Os problemas encontrados não podem ser associados a mais de uma classificação; M2.2 - Não houveram incertezas nos momentos de associar cada problema à sua classificação correta.

- OBJETIVO de AVALIAÇÃ̃̃ 2 - Análise: Método de Avaliação de OAs para a LDI; Para: Avaliação; Quanto à: Tempo de Avaliação; Na visão: Dos professores e alunos; No contexto: Da sala de aula; Objetivo 2: Verificar se o Método de Avaliação é eficiente quanto ao tempo; Questão 3: O tempo gasto para interpretar as classificações é o ideal, levando em consideração que uma avaliação heurística não deve ultrapassar duas horas Nielsen (1994)?; Métricas para realização da Questão 3: M3.1 - As classificações não estão redundantes ou muito breves, de forma em que não foi necessário muito tempo para interpretá-las; M3.2 - As palavras e/ou termos são de fácil compreensão; Questão 4:O tempo para concluir a avaliação foi ideal, levando em consideração que uma avaliação heurística deve ser realizada entre uma e duas horas Nielsen (1994)?; Métricas para realização da Questão 4: M4.1 -A avaliação não perdeu a qualidade devido a furação (muito longa e cansativa ou curta e superficial); M4.2 - Foi possível entender, identificar, executar, de de forma rápida cada passo do Método de Avaliação. 
VII Congresso Brasileiro de Informática na Educação (CBIE 2018)

Anais dos Workshops do VII Congresso Brasileiro de Informática na Educação (WCBIE 2018)

- OBJETIVO de AVAliAÇÃo 3 - Análise: Método de Avaliação de OAs para a LDI; Para: Avaliação; Quanto à: Problemas s/ classificação; Na visão: Dos professores e alunos; No contexto: Da sala de aula; Objetivo 3: Verificar se as classificações propostas pelo Método de Avaliação abrangem os problemas encontrados; Questão 5: Os problemas encontrados durante o processo de avaliação foram bem associados à suas respectivas classificações?; Métricas para realização da Questão 5: M5.1 - Sobraram poucos problemas (ou nenhum) sem uma classificação adequada; M5.2 -Todos os problemas foram classificados sem grandes incertezas; Questão 6: Os problemas que não foram classificados, são relevantes e afetam a usabilidade do OA?; Métricas para realização da Questão 6: M6.1 - Não, somente em caso muito específico; M6.2 - Não afetam.

É utilizado como representação da escala de avaliação das métricas referentes às questões 1, 2, 3, 4 e 5 e 6: (1) Discordo totalmente; (2) Discordo parcialmente; (3) Indiferente; (4) Concordo parcialmente; (5) Concordo totalmente. Caso mais da metade dos avaliadores concorde com as métricas utilizadas a avaliação se mostrará, pelo menos, um pouco eficiente.

\section{Resultados e Análise}

A média obtida pelo conjunto HOALDI é feita da seguinte maneira: calcula-se a média aritmética dos graus de severidade atribuídos pelos avaliadores, por cada heurística; calcula-se a média aritmética dos graus de severidade das heurísticas que pertencem a uma mesma categoria. Para o conjunto FCHECKLIST: calcula-se a porcentagem da unanimidade das respostas dadas pelos avaliadores, em cada pergunta da checklist; calcula-se a média aritmética as porcentagens das respostas que pertencem a uma mesma categoria.

A quantidade de classificações é dada a cada método de avaliação, pelo conjunto HOALDI e pela a FCHECKLIST. Ela foi organizada com as seguintes categorias: CE Carga Educacional; EE - Estratégia Educativa; MP - Manipulação; IF - Interface; ML - Manipulação. Em relação a quantidade de classificações por categoria dos conjuntos HOALDI e FCHECKLIST, de forma respectiva para HOALDI e FCHECKLIST, ficaram da seguinte maneira: $\mathbf{C E}-3$ e 1; EE - 5 e 9; MP - 2 e 3; IF -5 e 6; ML -7 e 5. As médias dos graus com base nessas classificações (Tabela 3) são:

Tabela 3. Médias dos graus de severidade das heurísticas dos conjuntos HOALDI e FCHECKLIST, obtidas por avaliadores especialistas e não especialistas

\begin{tabular}{|c|c|c|}
\hline \multicolumn{3}{|c|}{ FASE I - Não Especialistas } \\
\hline Categoria & $\begin{array}{c}\text { Grupo } \\
\text { HOALDI }\end{array}$ & $\begin{array}{c}\text { Grupo } \\
\text { FCHECKLIST(\%) }\end{array}$ \\
\hline $\mathrm{CE}$ & 0,66 & 50,00 \\
\hline $\mathrm{EE}$ & 0,80 & 44,44 \\
\hline MP & 1,50 & 33,33 \\
\hline IF & 1,20 & 50,00 \\
\hline ML & 0,86 & 40,00 \\
\hline Média & 1,00 & 43,55 \\
\hline \multicolumn{3}{|c|}{ FASE II - Especialistas } \\
\hline Categoria & $\begin{array}{c}\text { Grupo } \\
\text { HOALDI }\end{array}$ & $\begin{array}{c}\text { Grupo } \\
\text { FCHECKLIST }\end{array}$ \\
\hline $\mathrm{CE}$ & 0,66 & 100,00 \\
\hline $\mathrm{EE}$ & 1,00 & 77,77 \\
\hline MP & 2,00 & 66,66 \\
\hline IF & 1,00 & 66,66 \\
\hline ML & 0,71 & 80,00 \\
\hline Média & 1,07 & 78,22 \\
\hline
\end{tabular}


VII Congresso Brasileiro de Informática na Educação (CBIE 2018)

Anais dos Workshops do VII Congresso Brasileiro de Informática na Educação (WCBIE 2018)

Aplicou-se também um questionário para avaliar três fatores desses conjunto: Objetividade, Tempo de Avaliação, e Problemas s/ classificação. A eficiência das heurísticas e a dos fatores desse questionário são mostradas na Tabela 4 e 5, respectivamente.

\section{Tabela 4. Eficiência do Método de Avaliação utilizando os conjuntos HOALDI e FCHECKLIST (\%)}

\begin{tabular}{c|c|c} 
Categoria & EHOALDI (\%) & EFCHECKLIST(\%) \\
\hline CE & 100,00 & 50,00 \\
\hline EE & 80,00 & 57,14 \\
\hline MP & 75,00 & 50,00 \\
\hline IF & 83,33 & 75,00 \\
\hline ML & 82,56 & 50,00 \\
Média & 84,18 & 56,43 \\
\hline
\end{tabular}

Tabela 5. Eficiência para identificação de problemas de problemas dos conjuntos
HOALDI e FCHECKLIST (\%)
\begin{tabular}{|c|c|c|}
\hline Categoria & EHOALDI (\%) & EFCHECKLIST(\%) \\
\hline Objetividade & 93,54 & 63,60 \\
\hline Tempo de Avaliação & 91,05 & 69,09 \\
\hline Problemas s/ Classificação & 90,81 & 64,16 \\
\hline Média & 91,80 & 65,62 \\
\hline
\end{tabular}

\subsection{Teste de Hipóteses}

Os valores apresentados nos gráficos da Figura 2 provem indícios de que o conjunto HOALDI obteve maior eficiência, mas, apesar disso, faz-se necessário, por meio do teste de hipótese, realizar uma análise estatística dos dados, a fim de garantir uma confiabilidade maior das afirmações realizadas.

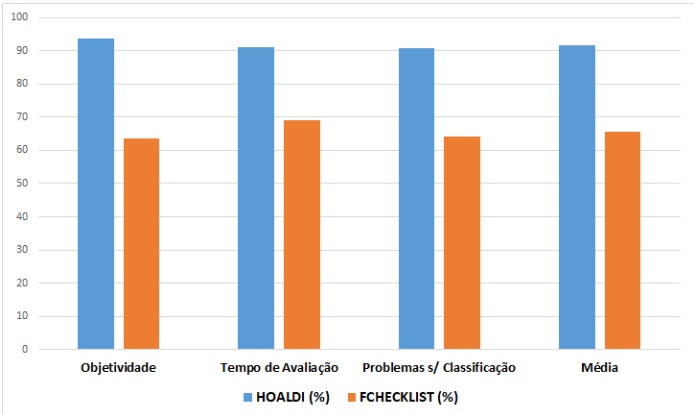

(a) Eficiência para identificação de problemas de problemas dos conjuntos HOALDI e FCHECKLIST (\%)

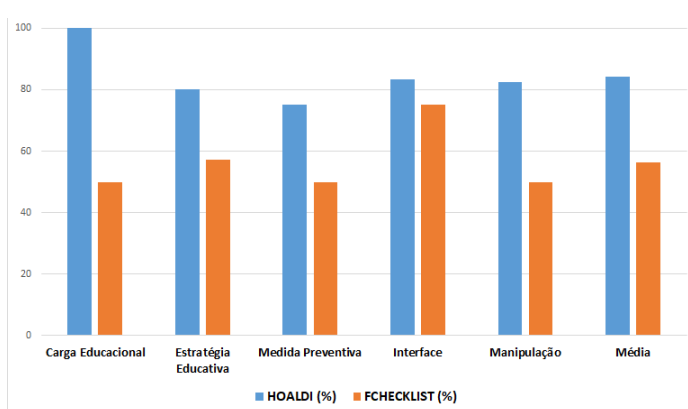

(b) Eficiência do Método de Avaliação utilizando os conjuntos HOALDI e FCHECKLIST (\%)

\section{Figura 2. Gráficos dos conjuntos HOALDI e FCHECKLIST}

O objetivo do teste de hipótese é verificar se H0 (hipótese nula), com algum grau de significância pode ser rejeitada , desta forma, chegando na aceitação da hipótese alternativa H1. Aplicou-se o teste t-student (Anderson e Whitcomb, 2000) ao conjunto de dados obtido, de forma a comparar os valores médios de eficiência dos grupos HOALDI e FCHECKLIST, apresentados na $\left(\mathrm{E}_{\text {нодеdi }}=84,18\right.$ е Е Еснескцігт $\left.=56,43\right)$. Utilizou-se, para os propósitos do presente estudo avaliativo, o menor grau de significância possível para a rejeição da hipótese nula. E também, além disso, ainda se utilizou o maior grau de significância aceito, neste trabalho, para a rejeição da hipótese nula foi de 5\%, sendo assim, a 
VII Congresso Brasileiro de Informática na Educação (CBIE 2018)

Anais dos Workshops do VII Congresso Brasileiro de Informática na Educação (WCBIE 2018)

hipótese nula só será levada em consideração caso possua um grau de confiabilidade igual ou maior a $95 \%$.

Aplicou-se o teste de normalidade Shapiro-Wilk Shapiro e Wilk (1965). Caso o valor da probabilidade de que seja rejeitado incorretamente a hipótese nula em favor da hipótese alternativa (W) for maior que a probabilidade da aceitação correta da hipótese nula, isso para um determinado grau de significância (p-value), então a hipótese nula é aceita e os dados são considerados que estão normalizados.

No presente trabalho, para o conjunto de valores da eficiência $\mathrm{E}_{\text {HодLdI }}=\{100,00$; $80,00 ; 75,00 ; 83,33 ; 82,56\}$, os dados foram considerados normalizados com grau de significância $\mathrm{p}=0,01$, pois $\mathrm{W}=0,86$ e Threshold $(\mathrm{p}=0,01)=0.68$. Para o conjunto Еғснескціsт $=\{50,00 ; 57,14 ; 50,00 ; 75,00 ; 50,00\}$, os dados também foram considerados normalizados com grau de significância $\mathrm{p}=0,01, \mathrm{~W}=0,716$ e Threshold $(\mathrm{p}=0,01)=$ 0.68 . Aplicando-se o teste t-student, com base nas médias das eficiências, Енодед $_{\text {H. }}=84,18$, e Eғснескціsт $=56,43$, a hipótese nula $\mathrm{H} 0$ pode-se rejeitar com grau de significância $\mathrm{p}=$ 0.0025 .

Com a análise dos resultados obtidos na avaliação dos testes feitos, foi possível refutar a hipótese nula e consequentemente verificar que há diferença entre as eficiências dos conjuntos analisados (HOALDI e FCHECKLIST). O conjunto HOALDI obteve eficiência de $84,18 \%$ e o conjunto FCHECKLIST, de 56,43\%. Após realizar os testes estatísticos, verificou-se, com $99 \%$ de confiabilidade, que o conjunto HOALDI possuiu uma melhor eficiência, quando comparado ao conjunto FCHECKLIST na identificação de problemas com avaliadores não são especialistas.

Logo, com aproximadamente 99\% de confiabilidade, pode-se afirmar que há diferença entre a eficiência para identificação de problemas em um OA, quando se utiliza o conjunto HOALDI ao invés do conjunto FCHECKLIST. Além disso, como a média da eficiência do Grupo HOALDI foi consideravelmente maior do que a do Grupo FCHECKLIST, há indícios de que o conjunto HOALDI é provavelmente mais adequado para avaliação de OAs, principalmente, quando avaliadores não especialistas são utilizados para estabelecer se o OA deve ser inserido em ambiente educacional ou não.

\section{Considerações finais e trabalhos futuros}

O conjunto HOALDI foi criado como um novo método de avaliação, de forma a aprofundar o estudo sobre LDI e qualidade de OAs. O mais importante do presente trabalho, é que avaliadores não especialistas poderão utilizar o conjunto HOALDI para desenvolver OAs e inseri-los no meio educacional, porque exige-se um certo esforço para encontrar avaliadores especialistas que entendam dos aspectos contemplados no conjunto HOALDI, ainda mais se tratando de uma tecnologia que tomou proporções grandiosas.

Como o cojunto HOALDI se trata de um método e avaliação genérico de OAs para a LDI, por meio de heurísticas, é interessante filtrar o tipo de OA que se deve avaliar, seja o foco para Ensino Fundamental ou Médio e qual área do conhecimento aplicar (como Biologia, Química, Matemática, etc), buscando particularidades nos objetivos e tornando o método de avaliação mais específico de acordo com a área.

Além disso, pretende-se avaliar um OA em outro contexto, com outro público, para verificar possíveis divergências entre as avaliações. De forma a mais tarde am- 
VII Congresso Brasileiro de Informática na Educação (CBIE 2018)

Anais dos Workshops do VII Congresso Brasileiro de Informática na Educação (WCBIE 2018)

pliar/modificar/alterar o conjunto HOALDI.

Um fator interessante a ser acrescentado ao conjunto HOALDI é a ergonomia. Não foram acrescentadas características específicas dos usuário, como idade e altura, isso acrescentaria muito ao conjunto HOALDI, visto que devido as particularidades da LDI o usuário se adapta melhor ou não.

\section{Referências}

Anderson, M. J. e Whitcomb, P. J. (2000). Design of experiments. Wiley Online Library. Audino, D. F. e da Silva Nascimento, R. (2010). Objetos de aprendizagem-diálogos entre conceitos e uma nova proposição aplicada à educação. Revista Contemporânea de Educação, 5(10).

Aytaç, T. (2013). Interactive whiteboard factor in education: Students' points of view and their problems. Educational Research and Reviews, 8(20), 1907.

Braga, J. C., Dotta, S., Pimentel, E., e Stransky, B. (2012). Desafios para o desenvolvimento de objetos de aprendizagem reutilizáveis e de qualidade. Em: Anais do Workshop de Desafios da Computação Aplicada à Educação (pp. 90-99).

de Castro Salgado, L. C., Bim, S. A., e de Souza, C. S. (2006). Comparação entre os métodos de avaliação de base cognitiva e semiótica. Em: Proceedings of VII Brazilian symposium on Human factors in computing systems (pp. 158-167).: ACM.

de Souza, F. M., da Cunha, A. M., e Torres, C. (2009). Uso do gqm para avaliar documentos de utilização de framework. Simpósio Brasileiro de Qualidade de Software.

Fiscarelli, S. H., Morgado, C. L., e Félix, M. A. (2016). Objetos de aprendizagem e lousas digitais interativas: uma proposta de avaliação de objetos de aprendizagem para ensino de matemática. Revista Ibero-Americana de Estudos em Educação, 11(25).

Kalinke, M. A. e Mocrosky, L. F. (2015). Educação matemática: pesquisas e possibilidades.

Mussoi, E. M., Flores, M. L. P., e Behar, P. A. (2010). Avaliação de objetos de aprendizagem. Em: Congresso Iberoamericano de Informática Educativa, Santiago, Chile. Anais.[Google Scholar].

Navarro, E. R. e Kalinke, M. A. (2016). Investigando o uso da lousa digital na rede estadual de ensino com o apoio de um curso de formação. Encontro Nacional de Educação Matemática, id. 7470_3840. SPEM, São Paulo.

Nielsen, J. (1994). Usability inspection methods. Em: Conference companion on Human factors in computing systems (pp. 413-414).: ACM.

Shapiro, S. S. e Wilk, M. B. (1965). An analysis of variance test for normality (complete samples). Biometrika, 52(3/4), 591-611.

Silva, H., Costa, D., Inocêncio, A. C., et al. (2017). Um mapeamento sistemático sobre o uso da lousa digital interativa no processo de ensino-aprendizagem. Em: Anais do Workshop de Informática na Escola, volume 23 (pp. 1203).

Silveira, M. S. e Carneiro, M. L. F. (2012). Diretrizes para a avaliação da usabilidade de objetos de aprendizagem. Em: Brazilian Symposium on Computers in Education (Simpósio Brasileiro de Informática na Educação-SBIE), volume 23.

Tarouco, L. M. R., da Silva, C. C. G., e Grando, A. (2011). Fatores que afetam o reuso de objetos de aprendizagem. RENOTE, 9(1). 